\title{
Increased intestinal permeability in rats with graft versus host disease
}

\author{
W A Koltun, M M Bloomer, P Colony, G L Kauffman
}

\begin{abstract}
Background/Aims-The study of graft versus host disease of the intestine has significant clinical relevance and may also be a model for other immune mediated intestinal diseases. There presently is no simple non-invasive test that can be used to evaluate graft versus host disease induced intestinal injury in humans or animal models. This study tested the hypothesis that graft versus host disease leads to an increase in host bowel permeability as assessed by the relative urinary excretion of orally administered lactulose and rhamnose.

Methods-The urinary excretion ratio of orally administered lactulose and rhamnose was determined daily for two weeks in (Lewis x Brown-Norway) F1 rats with graft versus host disease caused by either the transplantation of parental (Lewis) small bowel or the intraperitoneal injection of parental (Lewis) splenic lymphocytes.
\end{abstract}

Results-Significant twofold to fourfold increases in the lactulose to rhamnose ratio were seen in both small bowel transplant and splenic lymphocyte transfer animals suffering from graft versus host disease during the second postoperative week. This effect occurred sooner in small bowel transplant than in splenic lymphocyte transfer animals (postoperative day 7 versus 11 , respectively). The signs of graft versus host disease, including splenomegaly and altered intestinal mucosal architecture, as well as the increased lactulose to rhamnose ratio were significantly attenuated in small bowel transplant animals treated with cyclosporine A (10 mg/kg/day).

Conclusions-Graft versus host disease is associated with an increase in the lactulose to rhamnose clearance ratio reflecting an increase in host bowel permeability. This increase, along with the signs of systemic graft versus host disease, can be significantly ameliorated by cyclosporine $A$. The lactulose to rhamnose clearance ratio is a non-invasive technique that can be used to assess the intestinal effects of graft versus host disease and the associated increase in intestinal permeability.

(Gut 1996; 39: 291-298)

Keywords: graft versus host disease, intestinal permeability, small bowel transplantation, cyclosporine $\mathbf{A}$.
Graft versus host disease (GVHD) occurs in up to 75 per cent of patients after bone marrow transplantation and recently has also been described in small bowel transplant recipients. ${ }^{1-4}$ This condition results from the large number of lymphocytes present in both bone marrow and small bowel grafts that recognise the host tissue as foreign. ${ }^{5}$ In addition to the cutaneous effects such as alopecia and rash, GVHD commonly affects the intestine. An accurate diagnosis of GVHD can be difficult to establish when it affects the gastrointestinal tract because of its relatively non-specific symptomatology. Upper intestinal or rectal endoscopy and biopsy can aid in the diagnosis, but pose significant risks especially when performed repetitively to monitor disease progression. Similar difficulties are encountered in the experimental laboratory, where assessment of intestinal GVHD in animal models usually requires death of the animal. A minimally invasive test could be very useful in the appropriate clinical or experimental setting to diagnose intestinal GVHD or monitor response to treatment.

A relatively non-invasive method of assessing small bowel injury is the measurement of intestinal permeability using orally administered, non-metabolised, and minimally absorbed sugars, such as lactulose and rhamnose. Increases in the ratio of intestinal lactulose to rhamnose permeation and subsequent urinary clearance ( $L / R$ ) have been described in other immune mediated intestinal illnesses, such as Crohn's disease. ${ }^{6-8}$ It was hypothesised that a similar increase in $L / R$ would be seen in GVHD induced injury of the intestine. Such increases in intestinal permeability in GVHD after bone marrow transplantation have already been suggested using radioactive tracer techniques, but these studies have either been anecdotal or complicated by concurrent chemotherapy, which itself is known to increase bowel permeability. ${ }^{910}$ Small bowel transplantation has also been associated with increased intestinal permeability, but this has been in patients or animal models where the surgical manipulation of the intestine itself, malnutrition or simultaneous rejection phenomenon could have been responsible. ${ }^{11} 12$

We therefore undertook to study the $L / R$ clearance ratios in two different rat models of GVHD and in GVHD animals treated with cyclosporine A, to test the hypothesis that an increase in the $L / R$ urinary clearance ratio is associated with GVHD injury of the intestine. The present series of experiments used inbred rats in a sibling to parental cross to eliminate 


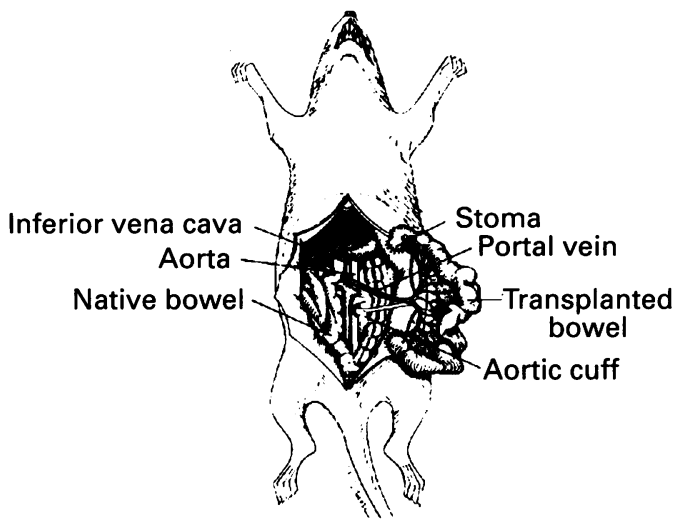

Figure 1: Small bowel transplant induced graft versus host disease animal model. Transplanted bowel (with passenger lymphocytes) is anastomosed in an auxiliary fashion to the recipient aorta and vena cava. The animal's native bowel is unmanipulated and has its permeability subsequently measured as graft versus host disease progresses.

the possibility of rejection and the native, nonoperated intestine was evaluated, so minimising the effects of surgical injury to the intestine. To characterise and confirm the presence and progression of GVHD in these models, food consumption, animal and splenic weights, and intestinal histology at the time of death was performed.

\section{Methods}

Animal models and groups

Male Lewis (Lew) and Lewis x Brown Norway (LBN) F1 virus antibody free rats $(250-300 \mathrm{~g})$ were obtained from a commercial breeder (Harlan Sprague Dawley, Indianapolis, IN) and handled in accordance with the guidelines of the American Association for Accreditation of Laboratory Animal Care. Animals were quarantined upon arrival from the supplier and monitored for one week for illness prior to use. Animals were housed in metabolic cages (0650-0100, Nalgene Co, Rochester, NY) during experimental protocol to permit easy collection of urine for bowel permeability assessment. Animals had free access to water and Purina Standard Rodent Chow 5001, although food was removed from cages during the hours of permeability measurements to avoid contamination of urine collections with food debris.

GVHD was produced by either the auxiliary transplantation of small bowel (Fig 1) or intraperitoneal injection of splenic lymphocytes from Lew (RT-1 ${ }^{1,1}$ ) donors into LBN F1 $\left(\mathrm{RT}-1^{1, \mathrm{n}}\right)$ hosts. Such a cross from inbred parent to $\mathrm{F} 1$ progeny produces GVHD without rejection because the major histocompatibility (MHC) antigens present in the homozygous grafted tissue are also present in the F1 recipient. The heterozygous $\mathrm{F} 1$ host however, expresses MHC antigens that are recognised as foreign by the grafted parental lymphocytes, resulting in GVHD. All animals, whether receiving small bowel grafts or intraperitoneal splenic lymphocytes, had their native intestines left intact for subsequent permeability measurements. Five groups of animals were studied:
(1) SBTx-GVHD ( $n=14)$ : LBNF1 animals with auxiliary Lew small bowel transplants (SBTx) resulting in GVHD;

(2) Sham-op controls $(\mathrm{n}=13)$ : LBNF1 animals with the same operative dissection as SBTX-GVHD animals but without small bowel grafts;

(3) Spl-GVHD ( $n=9):$ LBNF1 animals with an average of $3.9 \times 10^{8}$ (range 3.0$6.22 \times 10^{8}$ ) Lew splenocytes injected intraperitoneally, resulting in GVHD;

(4) Un-op controls $(n=8):$ LBNF1 animals without operative manipulation;

(5) SBTx-CsA $(n=8):$ LBNF1 animals with Lew small bowel transplants (SBTx) as in group 1 above who also received $10 \mathrm{mg} / \mathrm{kg} /$ day cyclosporine A (CsA) subcutaneously.

All animals had the permeability of their native (in situ) bowel assessed daily for two weeks after transplantation using the lactulose/ rhamnose $(\mathrm{L} / \mathrm{R})$ clearance technique (see below). Food cups were weighed daily to assess food consumption. Animals were weighed and inspected daily for signs of GVHD (cutaneous erythema, alopecia, weight loss, and dermatitis). Fourteen days after grafting or sham operation, animals were killed by exsanguination under ether anaesthesia. Spleens were weighed and segments of bowel harvested for histology to confirm the presence of intestinal GVHD.

\section{Technique of small bowel transplantation}

The technique of small bowel transplantation has been previously described. ${ }^{13}$ Briefly, small bowel from proximal jejunum to terminal ileum was harvested from the donor and subsequently anastomosed in an auxiliary fashion to the recipient's aorta and vena cava. The proximal and distal ends of the graft were brought out as stomas, creating an auxiliary Thiry-Villa loop. The animal's native bowel was left intact for subsequent bowel permeability assessment (Fig 1).

\section{Splenic lymphocyte transplantation}

Spleens were harvested from Lew rats using aseptic technique and placed into sterile Dulbecco's phosphate buffered saline (D-PBS, GIBCO BRL, Life Technologies, Grand Island, NY). Tissue was gently minced using forceps and the resultant suspension passed through a $70 \mu \mathrm{m}$ nylon mesh filter (Falcon, Becton Dickinson, Franklin Lakes, NJ). Red cells were lysed by the addition of $15 \mathrm{ml}$ of red cell lysing buffer (Sigma, St Louis, MO). Spleen cells were suspended with D-PBS in a $50 \mathrm{cc}$ conical tube and centrifuged for eight minutes at $200 \mathrm{~g}$ in a refrigerated centrifuge. The supernatant was discarded, the pellet washed two more times, and finally brought up to $10 \mathrm{cc}$ with D-PBS. A cell count was done using a haemocytometer and trypan blue exclusion to determine viability. An average of $3.9 \times 10^{8}$ viable cells (range 3.0 $6 \cdot 2 \times 10^{8}$ ) cells were injected intraperitoneally into recipient $\mathrm{LBN} F 1$ animals using an 18 gauge needle. It has been shown that a minimum of $1.5 \times 10^{8}$ splenocytes are necessary to cause GVHD in this rat model. ${ }^{14}$ 


\section{Lactulose/rhamnose clearance}

Animals were gavaged daily each evening with a solution containing $400 \mathrm{mg}$ lactulose and $100 \mathrm{mg}$ rhamnose (Pfanstiehl Laboratories, Waukegan, IL) in $5 \mathrm{cc}$ of distilled water. The urinary clearance of orally administered lactulose and rhamnose has been shown to be largely complete in rats by eight hours. ${ }^{15}$ Urine was collected for at least eight hours overnight in receptacles containing $200 \mu \mathrm{l}$ of chlorhexidine (Sigma, St Louis, MO) as a preservative. Urine samples were centrifuged at $200 \mathrm{~g}$ for 10 minutes and $1.0 \mathrm{ml}$ aliquots of the supernatant were frozen at $-70^{\circ} \mathrm{C}$ for later analysis by high pressure liquid chromatography (HPLC). Urine samples before gastric gavage were confirmed as containing unmeasurable amounts of both the test sugars and internal standard sugars (see below).

Stored urine specimens were defrosted on ice and centrifuged at $200 \mathrm{~g}$ for five minutes. The supernatant was diluted 10 -fold to $200-$ fold with deionised water to yield final test sugar concentrations that fell within the linear range of standard curves for each sugar. Internal standards, arabinose, and cellobiose (Pfanstiehl Labs, Waukegan, IL) were added to obtain a final concentration of $20 \mathrm{mg} / \mathrm{l}$. Samples were filtered through $0 \cdot 2 \mu$ pore size syringe tip filters (Supelco, Bellefonte, PA) and $20 \mu \mathrm{l}$ aliquots analysed by HPLC using an anion exchange column (Carbopac PAl, Dionex, Marlton, NJ) and pulsed amperometric electrochemical detection (Coulochem II, ESA, New Bedford, MA). ${ }^{6} 17$ Standard curves were run for test and internal standard sugars and confirmed to be linear by least squares analysis $\left(r^{2}>0 \cdot 95\right)$. Peak height analysis of the chromatograms (Spectra Physics SP 4270) was used to determine concentrations of the sugars. Test sugar results were expressed as the ratio: $L / R=$ percent urinary recovery of orally administered lactulose/percent urinary recovery of orally administered rhamnose.

\section{Absolute and relative spleen weights}

Both absolute and relative splenomegaly have been used as quantifiable indicators of GVHD. At the time of death (14 days postoperation) the absolute wet weight of each animal's spleen was determined to the nearest $0 \cdot 1 \mathrm{mg}$. One animal in the Spl-GVHD group died on day 13 so only eight animals in this group had spleen weights determined. Relative spleen weight was expressed as absolute spleen weight (g)/animal body weight at the time of death (kg).

\section{Intestinal histology}

To confirm the effects of GVHD on the host intestine, samples of small bowel were harvested at the time of death from eight Sham-op, eight SBTx-CsA animals, six SBTxGVHD animals (and three additional animals done subsequently for a total of nine SBTxGVHD animals), and six Spl-GVHD animals and evaluated histologically for crypt hyperplasia and villus atrophy. These two features have been shown to be indicative of intestinal GVHD. ${ }^{18-20}$ Full thickness segments of proximal jejunum, mid $(50 \mathrm{~cm})$ and distal $(90-100$ $\mathrm{cm}$ ) small bowel were harvested from each animal and stained in standard fashion with haematoxylin and eosin. An average villus and crypt length per segment of bowel studied was generated by a blinded histologist by measuring 20 well oriented villi and crypts with an eyepiece micrometer.

\section{Statistics}

Numerical data are expressed as mean (SEM).

Animal weight, food consumption, spleen and histological data were statistically evaluated using paired Student's $t$ test for comparisons within groups and unpaired Student's $t$ test for comparisons between groups. All $t$ tests were two tailed. Significance was considered achieved when $\mathrm{p} \leqslant 0 \cdot 05$.

The $L / R$ values from all the animals in each experimental group for each day were combined yielding a group mean and standard error for each day. It was noted that all animals who underwent operative manipulation (Sham-op, SBTX-GVHD, SBTx-CsA) had a transient increase in the $L / R$ ratio three to six days after surgery (see Fig 7). This was felt to be a side effect of the operation because it was absent in unoperated animals (Un-op, Spl-GVHD) and a similar effect has been noted in human studies. ${ }^{21} 22$ To eliminate this variable, a six day postoperative washout period was arbitrarily set and $L / R$ data were statistically compared only during the postoperative day 7-13 period (inclusive). This time period also coincided with the clinical manifestations of GVHD in the animals (see below). The $L / R$ data for this entire 7-13 day period for each group were then statistically compared using repeated measures analysis of variance as

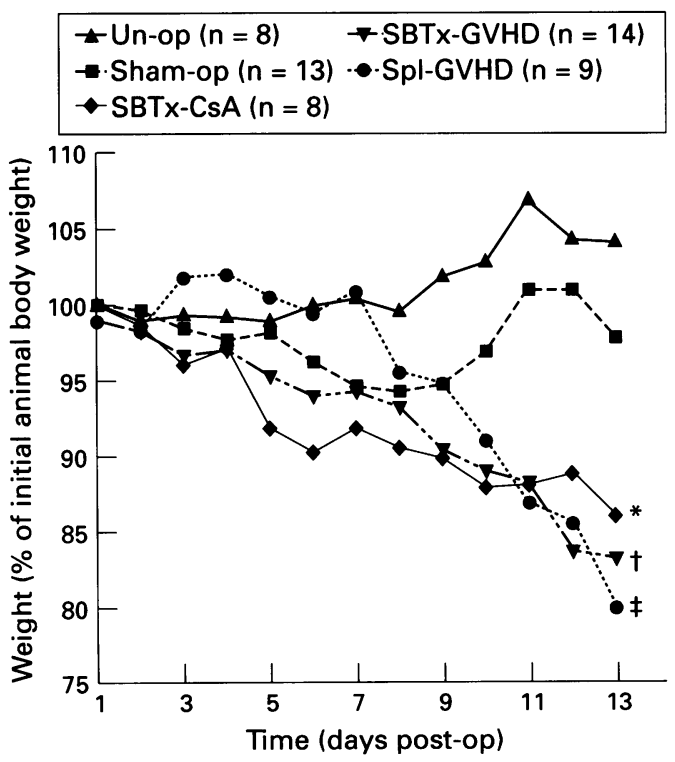

Figure 2: Mean weight for each animal group, expressed as per cent of initial weight. All experimental animal groups had lost a significant amount of weight by the time of death (14 days post-op) relative to sham or un-operated controls ${ }^{\star} p<0.05,+p<0.001 \mathrm{v}$ sham-op; $\neq p<0.001 \mathrm{v}$ un-op by unpaired Student's $t$ test). Standard error bars omitted for clarity. 


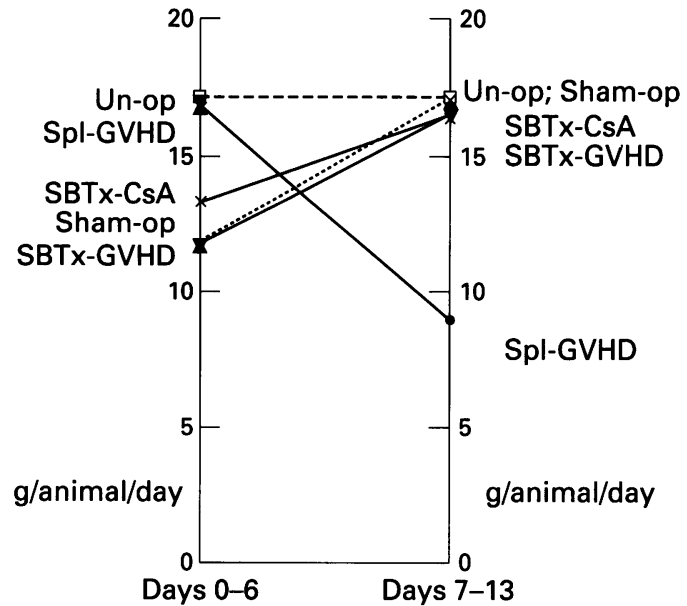

Figure 3: Average daily food consumption (grams/animal/day) for all animal groups for the first versus the second postoperative seven day periods. All animals undergoing surgery (sham-op, SBTx-GVHD, $S B T x-C s A)$ had significantly $(p<0.01)$ lower food consumption than un-operated animals (un-op, Spl-GVHD) during the first postoperative week. All animal groups had near identical food consumption during the second week, except for the Spl-GVHD group, which had a significant decrease $(p<0.001 \mathrm{v}$ all other groups and $\mathrm{v}$ day $0-6$, by unpaired and paired $\mathrm{t}$ test, respectively). Error bars eliminated for clarity.

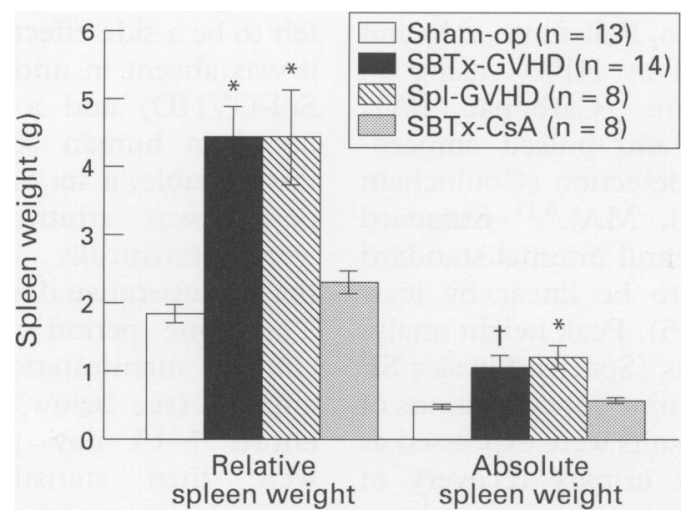

Figure 4: Mean relative spleen weights (left) and mean absolute spleen weights (right) in animal groups. Graft versus host disease was confirmed at death (14 days postop) in animals with small bowel transplants (SBTXGVHD) and splenocyte transfer (Spl-GVHD) by an increase in both relative spleen weight (absolute spleen weight (g)/body weight at death (kg)) and absolute spleen weight $(g) .\left({ }^{\star} p<0.001,+p<0.005 \mathrm{v}\right.$ sham-op by unpaired Student's $\mathrm{t}$ test). There was no statistical difference in either absolute or relative spleen weights between cyclosporine $A$ treated animals (SBTX-CsA) and sham-op controls.

implemented in SAS PROC GLM (SAS Institute, Cary, NC) and $p$ values reported.

\section{Results}

\section{Clinical appearance}

Both the SBTx-GVHD and Spl-GVHD animals recovered and appeared normal until the seventh or eighth postoperative day when the first signs of GVHD disease became manifest as mild cutaneous erythema of the paws, ears and snout, with some hair loss. These features gradually progressed to dramatic cutaneous hyperemia and dermatitis, alopecia, nasal and ocular discharge, and phimotic appearing
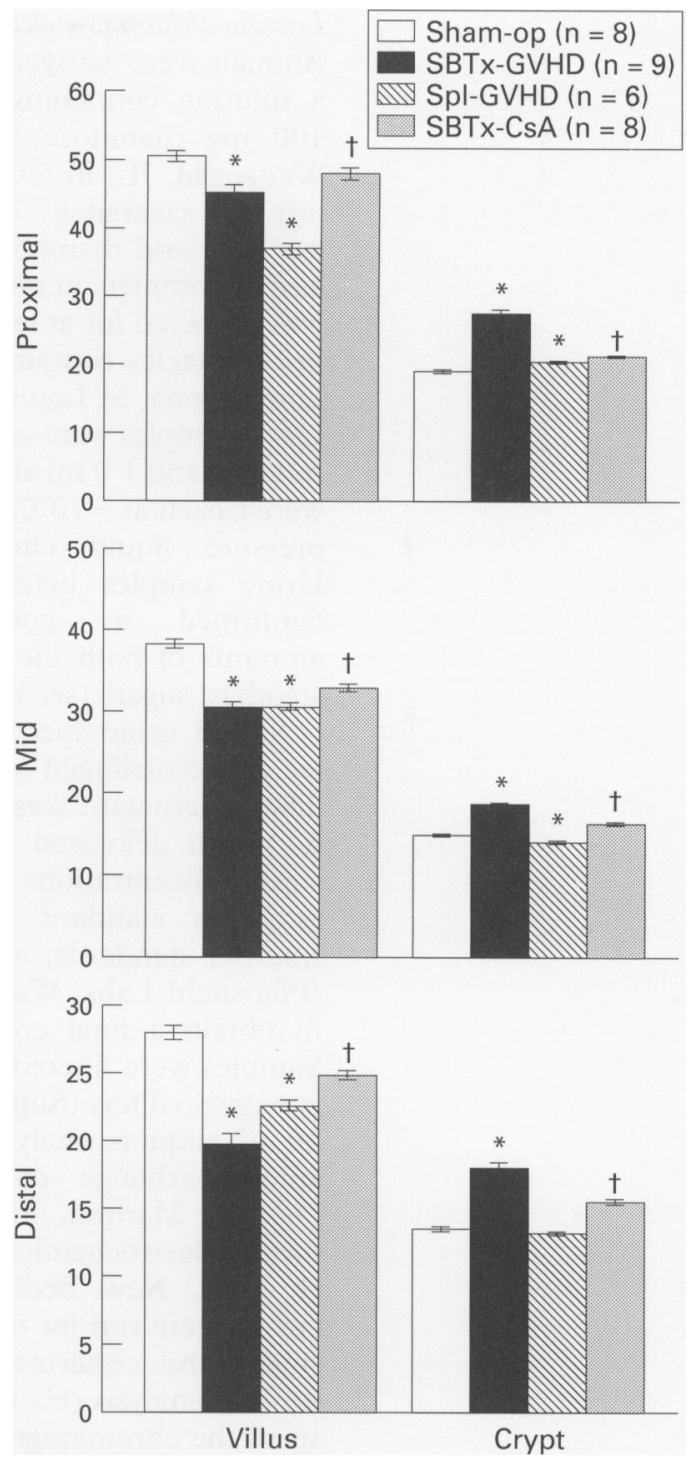

Figure 5: Villus height and crypt depth in proximal, mid, and distal small bowel in experimental groups of animals. Animals after SBTx (SBTx-GVHD group) had very significant decreases in villus height and increases in crypt depth in all regions of bowel studied compared with shamop animals, confirming the effects of GVHD on host intestine. All these changes improved significantly when animals were treated with cyclosporine $A$ (SBTx-Cs $A$ group) though they did not completely return to sham-op values. GVHD after splenic transplantation (Spl-GVHD group) also caused significant decreases in villus height but appeared to cause less severe crypt length changes when compared with that seen in the SBTx-GVHD animals $\left({ }^{\star} p\right.$ $<0.001$ versus respective sham-op group; $\dagger p<0.01$ versus respective $S B T x-G V H D$ group).

genitalia. There was no diarrhoea. Stools became viscid, but were always in pellet form, a fortuitous situation as urine collections were not contaminated by liquid faecal material. Animals became less active and assumed a hunched posture with apparent difficulty in fully extending their limbs by the 13 th to 14 th day. Sham operated animals recuperated and returned to normal activity and appearance promptly without clinical deterioration during the experimental period. Unoperated animals exhibited normal activity and behaviour. SBTx-CsA animals appeared generally healthy even during the second postoperative week. Mild cutaneous erythema was observed in some, 


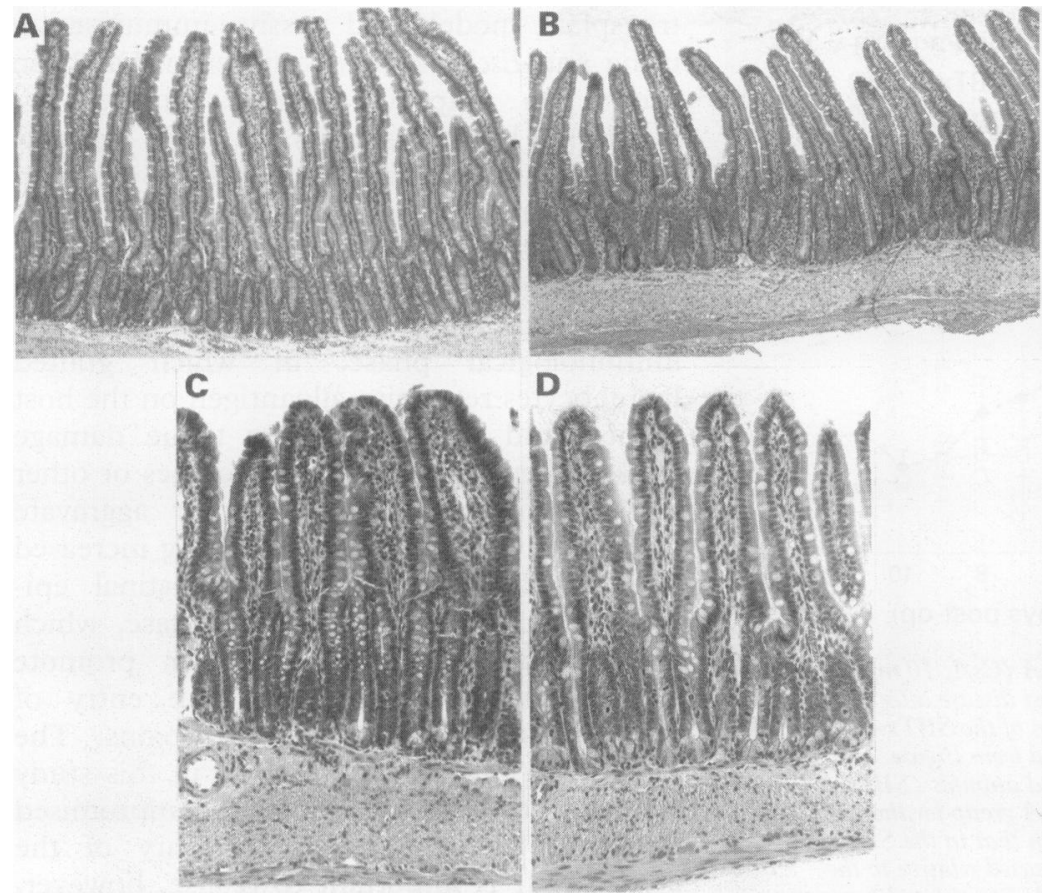

Figure 6: Proximal small bowel micrographs from sham-op $(A)$ and $S B T x-G V H D(B)$ animals (original magnification $\times 125$ ). Higher power micrographs (original magnification $\times 312$ ) of distal small bowel from sham-op(C) and $S B T x-G V H D(D)$ animals. Note villus blunting and crypt elongation in GVHD specimens compared with sham-op. Intestinal epithelial integrity is maintained without evidence of ulceration or increased apoptosis in the GVHD animals.

however, there were no hair or mucous membrane changes.

\section{Food consumption/weight changes}

Figure 2 shows the mean weight change of the animal groups over the course of the experiment. The Un-op group gained an insignificant amount of weight during the two week period, while the Sham-op group had a

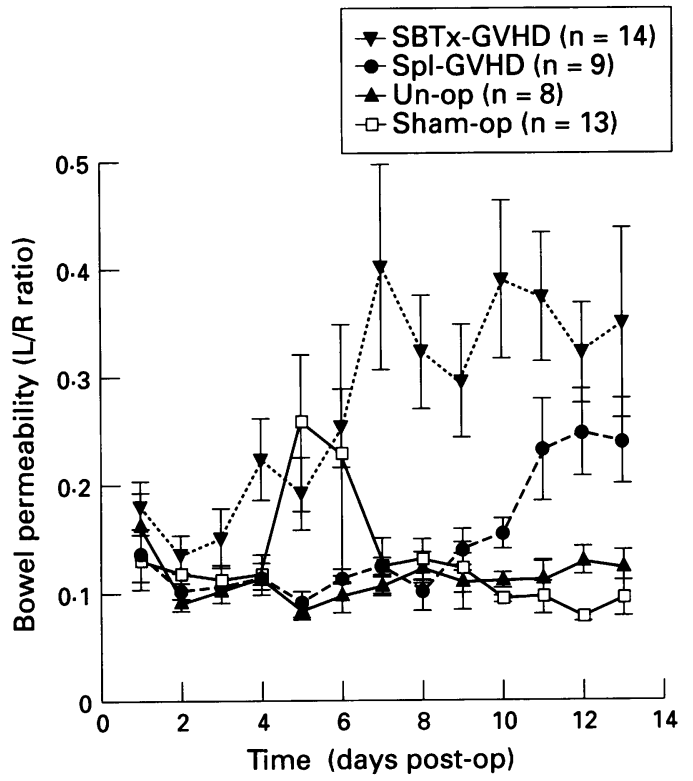

Figure 7: Bowel permeability as defined by $L / R$ ratios in controls (sham-op and un-op) and animals with graft versus host disease after small bowel transplantation (SBTx-GVHD) or after splenic lymphocyte transfer (SplGVHD). For days $7-13, L / R$ was significantly increased in SBTx-GVHD animals $\mathrm{v}$ sham-op controls $(p<0 \cdot 001)$ and in Spl-GVHD animals v un-op controls $(p<0.02)$ by repeated measures analysis of variance. The overall increase in $L / R$ in the SBTx-GVHD group for the 7-13 day period was statistically greater than that in the Spl-GVHD group at the $p=0.05$ level. temporary period of postoperative weight loss before returning to a weight comparable to the preoperative level. Animals with either form of GVHD, as well as the CsA treated animals all had a similar pattern of weight loss over the experimental period that resulted in these three groups having a final weight at death significantly less than Sham-op or Un-op controls.

Figure 3 shows the mean daily food consumption for each of the groups during the first and second 7 day postoperative periods of the experiment. Food consumption during the first week was very similar, but significantly depressed in those animal groups that underwent surgery compared with those animal groups that did not have surgery. Food consumption during the second week returned to control levels in all animal groups, except the Spl-GVHD group in which there was a significant decline. This decrease in food intake may have accounted, in part, for the concurrent rapid weight loss seen in this animal group. The weight loss seen in the SBTx-GVHD and SBTX-CsA groups during the second week, however, could not be attributed to decreased dietary intake.

\section{Spleen weights}

Relative and absolute spleen weights confirmed the presence of GVHD in the experimental groups and the efficacy of CsA treatment in treating GVHD in animals with small bowel transplants (Fig 4).

\section{Intestinal histology}

There were significant reductions in villus height and increases in crypt depth in SBTxGVHD animals compared with Sham-op controls in the proximal, mid, and distal regions of the small bowel confirming the effects of GVHD on the host intestine (Fig 5). In SBTx-GVHD animals, villus blunting appeared to be most severe in the distal bowel where mean villus height was $38 \%$ less than Sham-op controls whereas it was only $12 \%$ and $20 \%$ less in the proximal and mid small bowel areas, respectively. Though there was also significant villus blunting diffusely in Spl-GVHD animals, crypt elongation was less prominent, suggesting less severe intestinal injury compared with SBTxGVHD animals. It is noteworthy that epithelial integrity was maintained in all segments of bowel studied in both GVHD animal groups with no loss of surface epithelium or ulcerations noted (Fig 6). When SBTx animals were treated with CyA (SBTx-CyA group), there were significant improvements in villus and crypt lengths compared with SBTX-GVHD animals, approaching though not completely returning to Sham-op control values in all segments of intestine studied.

\section{Lactulose/rhamnose bowel permeability}

Unoperated control animals (Un-op group) showed very consistent mean $L / R$ ratios from day to day during the two week experimental periods (Fig 7). Sham-op L/R ratios were 


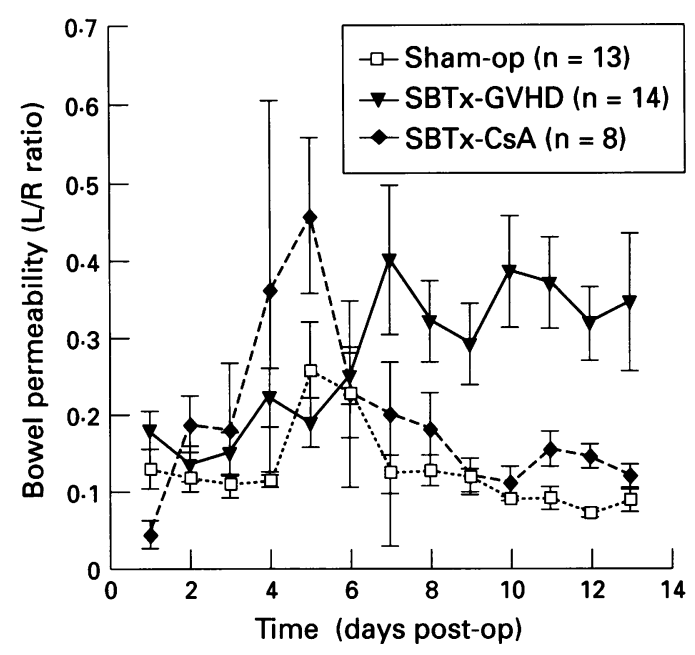

Figure 8: Effect of cyclosporine $A$ (Cs $A, 10 \mathrm{mg} / \mathrm{kg} /$ day) on $L / R$ in rats with graft versus host disease after small bowel transplantation. The $L / R$ curves of the SBTX-GVHD and sham-op animals are reproduced from Figure 7 for comparison with the CsA treated animals ( $B B T x-C s A)$. The $L / R$ ratios of the $S B T x-C s A$ group for the 7-13 day period was significantly less than that in the $S B T x-G V H D$ group $(p<0.025)$, but still increased relative to the sham-op control group $(p<0.04)$ by repeated measures analysis of variance.

statistically no different than those of the Un-op group during days 7-13, although there appeared to be a transient increase in $L / R$ at days 5 and 6 as previously mentioned.

Compared with the Sham-op group, the SBTx-GVHD group exhibited a statistically significant increase in $L / R$ ratios for the 7-13 day period $(p<0.001)$, when the clinical signs of GVHD were manifest. Similarly, there was a statistically significant increase in the $L / R$ ratio in the GVHD-Spl animals versus Un-op animals during the same 7-13 day period $(p<0.02)$. Qualitatively, the increase in the L/R ratio in the SBTx-GVHD group occurred earlier than that seen in the Spl-GVHD group (postoperative day 7 versus postoperative day 11 , respectively). When these two groups were statistically compared, this difference was found to be significant ( $\mathrm{p}=0.05, \mathrm{GVHD}-\mathrm{SBT} x$ $v$ GVHD-Spl).

Figure 8 shows the L/R ratios of the SBTxCsA group compared with the SBTx-GVHD and Sham-op groups. When animals receiving small bowel transplants were treated with CsA (SBTx-CsA group), L/R ratios for the 7-13 day period were significantly lower than that seen in the untreated SBTx-GVHD group $(p<0.025)$. Cyclosporine A did not completely ameliorate the raised $L / R$ ratios seen in the SBTx-GVHD group however, because ratios in the SBTx-CsA group were still significantly increased relative to the Sham-op group $(\mathrm{p}<0.04)$.

\section{Discussion}

The concept of the gut as a reservoir of bacteria or toxins that aggravates local injury or leads to systemic illness when the protective enteric permeability barrier is compromised, is one that has been proposed in numerous illnesses, including GVHD. ${ }^{23-26}$ Sterilisation or selective decontamination of the gut has attenuated GVHD in murine bone marrow transplant models and passive immunisation using anti-Escherichia coli antiserum has been shown to improve animal survival. ${ }^{24-27}$ Similarly, human studies in bone marrow transplant recipients have suggested that gut decontamination with oral antibiotics may decrease the clinical signs and infectious complications of GVHD. ${ }^{28} \mathrm{~A}$ two stage model of intestinal injury in GVHD includes an immunological phase, in which grafted lymphocytes recognise alloantigen on the host tissues, and an effector or tissue damage phase, where the release of cytokines or other inflammatory mediators incur or aggravate injury to the bowel. ${ }^{25}$ The resulting increased permeability of the injured intestinal epithelium may represent a third phase, which might augment local injury or promote systemic sepsis by allowing the entry of noxious luminal microflora or toxins. The increase in the $L / R$ ratio found in this study supports this concept of a compromised intestinal barrier in GVHD injury of the intestine. It is important to realise, however, that this increase in bowel permeability as identified by the raised $L / R$ ratio need not necessarily correlate with increased bowel permeability to other compounds, including enteric organisms. Different mechanisms for uptake of various materials exist in the intestine. Translocation of bacteria and fungi has been shown to occur transcellularly, for example. ${ }^{29}$ The presently identified increase in L/R therefore, cannot be freely extrapolated to other materials, including much larger substances such as endotoxin or bacteria but does reinforce the notion that the intestinal defensive barrier is compromised in GVHD.

This study suggests increases in $L / R$ are a consequence of immune mediated inflammation directed towards the intestine during GVHD. The exact mechanism is unclear but may entail the local release of potent cytokines. $^{30}$ Two cytokines that have been implicated in GVHD injury include interferon $\gamma$ (IFN $\gamma$ ) and tumour necrosis factor (TNF). Increased serum concentrations of TNF in GVHD after SBTx have been described and antibodies to both IFN $\gamma$ and TNF have been shown to ameliorate some of the histopathological changes of GVHD injury in mice. ${ }^{14} 1831$ Of significant interest, IFN $\gamma$ has also been shown to play a part in intestinal barrier function. Ussing chamber studies using cultured monolayers of $\mathrm{T}-84$ human intestinal cells have shown a decrease in transepithelial resistance at the level of the intercellular tight junction after IFN $\gamma$ administration. ${ }^{32} 33$ As the L/R technique of bowel permeability measurement is felt to assess paracellular integrity, the alteration defined in this study may represent the in vivo correlate of this in vitro increase in epithelial permeability that occurs secondary to the local release of this cytokine during GVHD intestinal injury.

Weight loss during GVHD has been described before and may result from a number of factors, including intestinal dysfunction, compromised ability to utilise the absorbed nutrients or a hypermetabolic state. ${ }^{34}$ 
It is noteworthy that there was a similar pattern of weight loss in the SBTx-CsA animals who also had control levels of food intake but did not manifest overt signs of GVHD. This suggests that either CsA itself had cachexia inducing effects, or that CsA imperfectly treated the GVHD. The latter is probably the case as firstly, the $L / R$ ratios did not return completely to control values in the SBTx-CsA group and secondly, a subsequent group of six animals injected daily with CsA $(10 \mathrm{mg} / \mathrm{kg})$ had a mean weight after 14 days virtually identical to their mean starting weight (103 $(3.5) \%$ ). The weight loss seen in the SBTxCsA animals also disputes the possibility that the significant increases in the $L / R$ ratios seen in the GVHD groups of animals were simply due to generalised nutritional deterioration causing intestinal atrophy and injury, because the SBTx-CsA group had a relatively low L/R ratio associated with significant weight loss.

The increased $L / R$ ratio seen in this study was significant and presents the sugar clearance technique as a comparatively noninvasive method by which to follow GVHD injury in the controlled laboratory or clinical setting. Assessment of treatment directed at ameliorating intestinal GVHD injury could be accomplished, in part, by repetitive measurement of the $\mathrm{L} / \mathrm{R}$ clearance ratio. Such a study in Crohn's disease patients showed an increased lactulose/rhamnose ratio in those with active disease, which decreased when patients were treated and became clinically improved. ${ }^{6}$ It would be tempting to suggest that the lactulose/rhamnose measurement could provide a non-invasive method by which to diagnose intestinal GVHD in patients undergoing bone marrow or small bowel transplantation. Limitations on the applicability of the technique may exist, however, as many other factors have been associated with increased bowel permeability. These include surgery, chemotherapy, neoplasia, sepsis, and malnutrition, many of which could also be present in the patient with GVHD. ${ }^{23} 35-37$ Repetitive $L / R$ measurements may none the less be clinically useful in the individual GVHD patient by providing an improving or deteriorating trend that could minimise the need for more morbid endoscopic biopsy procedures. In this regard, the $L / R$ technique has significant advantages over other techniques of bowel permeability measurement, especially those using radioactive tracers, as it can be repetitively applied in the clinical arena with minimal risk. ${ }^{10}$ In the controlled laboratory setting, the $L / R$ ratio could similarly be effective. Therapeutic interventions aimed at decreasing intestinal GVHD injury, such as was done here with CsA, could be assessed in animals on a daily basis without the need for death.

The clinical manifestations of GVHD in the SBTx-GVHD and the Spl-GVHD groups were similar, but both the $\mathrm{L} / \mathrm{R}$ and intestinal histology data suggest that a more severe intestinal injury occurred in the SBTx-GVHD animals. Crypt elongation occurred in proximal, mid, and distal small bowel in the
SBTx-GVHD animals, but was present only in the proximal portions of the bowel in SplGVHD animals. The L/R increase appeared sooner in SBTx-GVHD animals compared with Spl-GVHD animals (day $7 v 11$, respectively). The disparity in food consumption between the Spl-GVHD and SBTx-GVHD animals during the second week also suggest that GVHD after SBTx may be different than that seen with splenocyte transfer. Such differences may be related to the finding that lymphocytes from the intestine tend to 'home' back to the gut. ${ }^{38}$ GVHD intestinal injury after SBTx may be greater than that caused by peripheral or splenic lymphocyte transfer because of this trafficking phenomenon, directing and preferentially localising the donor enteric lymphocytes and injury to the host bowel. This suggests that data regarding GVHD mediated intestinal injury obtained from clinical bone marrow transplantation patients may not be directly applicable to the increasing number of patients receiving small bowel transplants. Further clinical and animal studies are warranted, evaluating this concept of lymphocyte homing and host intestinal injury after bowel transplantation.

In summary, using both splenocyte and small bowel transplant models of GVHD, increases in host bowel permeability, as measured by the lactulose/rhamnose urinary clearance technique were observed. This increase in the lactulose/rhamnose ratio was attenuated by treatment of small bowel transplant animals with cyclosporine A. These data suggest that the lactulose/rhamnose urinary clearance assay may provide a non-invasive method by which to assess intestinal injury and the effect of treatment in GVHD mediated injury of the bowel.

1 Brubaker DB. Immunopathogenic mechanisms of posttransfusion graft-vs-host disease. Proc Soc Exp Biol Med 1993; 202: 122-47.

2 Grant D, Garcia B, Wall W, Oheme-Fianko D, Zhong R Mimeault R, et al. Graft-versus-host disease after clinical small bowel/liver transplantation. Transplant Proc 1990; 22: 2464 .

3 Cohen Z, Silverman RE, Wassef R, Levy GA, Burnstein M, Cullen J, et al. Small intestinal transplantation using cyclosporine. Transplantation 1986; 42: 613-21.

4 Wick MR, Moore SB, Gastinear DA, Hoagland HC mmunologic, clinical and pathologic aspects of human graft-versus-host disease. Mayo Clin Proc 1983; 58: 603-12.

5 Pirenne J. Study on mechanisms of graft-versus-host disease following small bowel transplantation. Acta Gastroenterol Belg 1992; LV: 17-22.

6 Sanderson IR, Boulton P, Menzies I, Walker-Smith JA. Improvement of abnormal lactulose/rhamnose permeability in active Crohn's disease of the small bowel by an eleity in active Crohn's disease of the

7 Pearson ADJ, Eastham EJ, Laker MF, Craft AW, Nelson R. Intestinal permeability in children with Crohn's disease and coeliac disease. BMf 1982; 285: 20-1.

8 Bjarnason I. Intestinal permeability. Gut 1994; 35 (Suppl 1): $\$ 18-22$.

9 Selby P, McElwain TJ, Crofts M, Lopes N, Mundy J. 51CrEDTA test for intestinal permeability. Lancet 1984; ii: 38-9.

10 Mahendra P, Bedlow AJ, Ager S, Ancliff PJ, Wraight EP, Marcus RE. Technetium( $(99 \mathrm{mTc})$-labelled white cell scanning, 51 CR-EDTA and 14C-mannitol-labelled intestinal permeability studies: non-invasive methods of diagnosing acute intestinal graft-versus-host disease. Bone diagnosing acute intestinal graft-vers

11 Grant D, Hurlbut D, Zhong R, Wang P, Chen H, Garcia B, et al. Intestinal permeability and bacterial translocation et al. Intestinal permeability and bacterial translocation Transplantation 1991; 52: 221-4.

12 Sigalet DL, Kneteman NM, Fedorak RN, Kizilisik AT, Thomson AB. Intestinal function following allogenic small intestinal transplantation in the rat. Transplantation 1992; 53: 264-71.

13 Koltun WA, Diamantstein T, Kirkman RL. Synergism between anti-interleukin-2 receptor monoclonal antibody 
and cyclosporine in small bowel transplantation. Curr Surg 1987; 44: 315-8.

14 Pirenne J, Degiovanni G, Franchimont N, Scheppens G Meager T, Dunn DL. Graft vs host disease after small bowel transplantation or lymphocyte transfer is associated with tumor necrosis factor alpha in the serum. Surg Forum 1990; 49: 389-91.

15 Hamilton I, Rothwell J, Archer D, Axon TR. Permeability of the rat small intestine to carbohydrate probe molecules. Clin Sci 1987; 73: 189-96.

16 Fleming SC, Kapembwa MS, Laker MF, Levin GE, Griffin GE. Rapid and simultaneous determination of lactulose and mannitol in urine, by HPLC with pulsed amperometric detection, for use in studies of intestinal permeability. Clin Chem 1990; 36: 797-9.

17 Rocklin RD, Pohl CA. Determination of carbohydrates by anion exchange chromatography with pulsed amperometric detection. Fournal of Liquid Chromatography 1983; 6: ric detection
$1577-90$.

18 Mowat AM. Antibodies to IFN- $\gamma$ prevent immunologically mediated intestinal damage in murine graft-versus-host reaction. Immunology 1989; 68: 18-23.

19 MacDonald TT, Ferguson A. Hypersensitivity reactions in the small intestine. The effects of allograft rejection and of graft-versus-host disease of epithelial cell kinetics. Cell Tissue Kinet 1977; 10: 301-12.

20 Mowat AM, Ferguson A. Intraepithelial lymphocyte count and crypt hyperplasia measure the mucosal component of the graft-versus-host reaction in mouse small intestine. Gastroenterology 1982; 83: 417-23.

21 Ohri SK, Somasundaram S, Koak Y, Macpherson A, Keogh BE, Taylor KM, et al. The effect of intestinal hypoperfusion during cardiopulmonary bypass surgery on hypoperfusion during cardiopulmonary bypass surgery on saccharide permeation and intestinal
Gastroenterology 1994; 106: 318-23.

22 Roumen RM, van der Vliet JA, Wevers RA, Goris RJ. Intestinal permeability is increased after major vascular surgery. F Vasc Surg 1993; 17: 734-7.

23 Deitch EA. The role of intestinal barrier failure and bacterial translocation in the development of systemic infection and multiple organ failure. Arch Surg 1990; 125: 403-4.

24 Lampert IA, Moore RH, Huby R, Cohen J. Observations on the role of endotoxin in graft-versus-host disease. In: Levin J, ed. Bacterial endotoxins: pathophysiological effects, clinical significance, and pharmacological control. New York: clinical significance, and pharm

25 Faulkner L, Rapson N, Moore R, Cohen J. The influence of the gut flora on graft versus host disease (GVHD) following allogeneic bone marrow transplantation - experimental observations and possible mechanisms. In: Levin J, ed. Bacterial endotoxins: pathophysiological effects, clinical significance, and pharmacological control. New York: Alan R Liss, 1988: 195-206.

26 Van Bekkum DW, Knaan S. Brief communication. Role of bacterial microflora in development of intestinal lesions from graft-versus-host reaction. Natl Cancer Inst Monogr from graft-versus-hos

27 Keast D, Walters MNI. The pathology of murine runting and its modification by neomycin sulphate gavages. Immunology 1968; 15: 247-62.

28 Schmeiser T, Kurrle E, Arnold R, Heit W, Krieger D, Kubanek $B$, et al. The influence of the microbial flora on incidence of graft versus host disease in human allogeneic bone marrow transplantation. In: Wostmann BS, Pleasants JR, eds. Germfree research: microflora control and its application to the biomedical sciences. New York: Alan R Liss, 1985: 433-5.

29 Alexander JW, Boyce ST, Babcock GF, Gianotti L, Peck MD, Dunn DL, et al. The process of microbial translocaMD, Dunn DL, et al. The process of
tion. Ann Surg 1990; 212: 496-512.

30 Elson CO, Reilly RW, Rosenberg IH. Small intestinal injury in the graft versus host reaction: an innocent bystander phenomenon. Gastroenterology 1977; 72: 886-9.

31 Piguet PF, Grau GE, Allet B, Vassalli P. Tumor necrosis factor/cachectin is an effector of skin and gut lesions of the acute phase of graft-vs-host disease. 7 Exp Med 1987; 166: 1280-9.

32 Adams RB, Planchon SM, Roche JK. IFN- $\gamma$ modulation of epithelial barrier function. Time course, reversibility, and
site of cytokine binding. $\mathcal{f}$ Immunol 1993; 150: $2356-63$.

33 Madara JL, Stafford J. Interferon- $\gamma$ directly affects barrier function of cultured intestinal epithelial monolayers. function of cultured intestinal

34 Hedberg CA, Reiser S, Reilly RW. Intestinal phase of the runting syndrome in mice. Transplantation 1968; 6: $104-10$

35 Elia M, Goren A, Behrens R, Barber RW, Neale G. Effect of total starvation and very low calorie diets on intestinal permeability in man. Clin Sci 1987; 73: 205-10.

36 Rhodes RS, Karnovsky MJ. Loss of macromolecular barrier function associated with surgical trauma to the intestine. Lab Invest 1971; 25: 220-9.

37 Penn RL, Maca RD, Berg RD. Increased translocation of bacteria from the gastrointestinal tracts of tumor-bearing mice Infect Immun 1985; 47: 793-8.

38 Chin W, Hay JB. A comparison of lymphocyte migration through intestinal lymph nodes, subcutaneous lymph nodes, and chronic inflammatory sites of sheep. Gastroenterology 1980; 79: 1231-42. 